\title{
Research on Analysis Method of Shading and Blocking of LFR Field
}

\author{
Jun $\mathrm{Ma}^{1,2}$, Chenglong Wang ${ }^{1, *}$ and Yangjun $\mathrm{Xia}^{2}$ \\ ${ }^{1}$ National Engineering Research Center for Technology and Equipment of Environmental Deposition, Lanzhou Jiaotong University, \\ Lanzhou, Gansu 730070, China \\ ${ }^{2}$ Key Laboratory of Opto-Technology and Intelligent Control of Ministry Education, Lanzhou Jiaotong University, Lanzhou, Gansu \\ 730070, China
}

\begin{abstract}
Shading and blocking problem is the main element restricting efficiency of a LFR system. The traditional analysis methods often need to build the models by using SolTrace, which results in the low efficiency. According to characteristics of single axis tracking of the LFR field, a plane right-angle coordinate system was established, and coordinate equations of reflectors at any time were deduced. Lengths of shading and blocking were got by tracking sun rays which fell on the reflectors and reflected the receiver passing through the adjacent reflector endpoints at any time. Offset caused by the solar azimuth in the shading and blocking area was got by geometrical optics. And then, the shading and blocking efficiency could be calculated. For the Puerto Errado 1 solar thermal plant, the shading and blocking efficiencies on vernal equinox hardly changed; on summer solstice, they were smaller at sunrise and sunset, and gradually stable and close to the value of spring equinox with the increase of solar altitude angle; they were smaller on winter solstice and the change with time were larger. The results of the calculations were good agreement with the simulation dates of SolTrace, and validity of the method was proved.
\end{abstract}

\section{Introduction}

As the most abundant renewable energy resource, solar energy has gained the interest of scientists all over the world [1-3]. In order to increase its efficient use, collectors that maximize sun power density are developed. According to concentration type, solar collector system mainly includes parabolic trough collector, linear Fresnel reflector (LFR), central receiver and parabolic dish etc. [4-7]. The LFR system is gradually being large-scale applications because of its simple construction and low wind loads $[8,9]$.

For the LFR system, optical efficiency, which is the chief basis of designing concentrator system of a solar thermal plant, is greatly limited by the shading and blocking between adjacent reflectors. Therefore, it is critical to accurately analyze shading and blocking efficiency of the LFR system.

Many studies have focused on the optical performance characterization of LFRs by optical modelling methods [10-12]. So, an analytical method of shading and blocking should be developed. Budin et al. [13] developed the mathematical model for shading calculations and derived the closed-form expressions for shading position of an isolated point on a plane surface with arbitrary orientation. However, the boundary conditions of shading and blocking analysis were not easy to judge. And they were liable to cause repeated calculation. Niewienda et al. [14] suggested the PC- program wrote in Turbo-Pascal to calculate the proportion of the shaded area of an arbitrarily oriented surface surrounded by shading elements. But targeted research of shading and blocking of the LFR system had not been progressed substantially and it has a large amount of calculation. Du et al. [15] got shading and blocking distribution of analyzed mirror element and analyzed annual average shading and blocking efficiency of the LFR field by Statistic means. But it was difficult to set up a global coordinate for vector analysis. Moreover, it was necessary to divide the reflectors into smaller units. The units were larger, resulting in large calculation errors. On the contrary, the computation was so big.

According to characteristics of single axis tracking of the LFR field, a plane right-angle coordinate system was established, and coordinate equations at any time of reflectors were deduced. With ray tracing method, lengths of shading and blocking were got by tracking sun rays which fell on the reflectors and reflected the receiver passing through the adjacent reflector endpoints at any time. Offset caused by the solar azimuth in the shading and blocking area was got by geometrical optics. And then, the shading and blocking efficiency of the LFR field could be calculated. The results of the calculations were good agreement with the simulation dates of SolTrace, and validity of the method was proved.

\section{Coordinate system of LFR field}

\footnotetext{
*Corresponding author: clwang@mail.lzjtu.cn
} 
A plane right-angle coordinate system with the origin at the center of a LFR field is established, as shown in Figure 1.

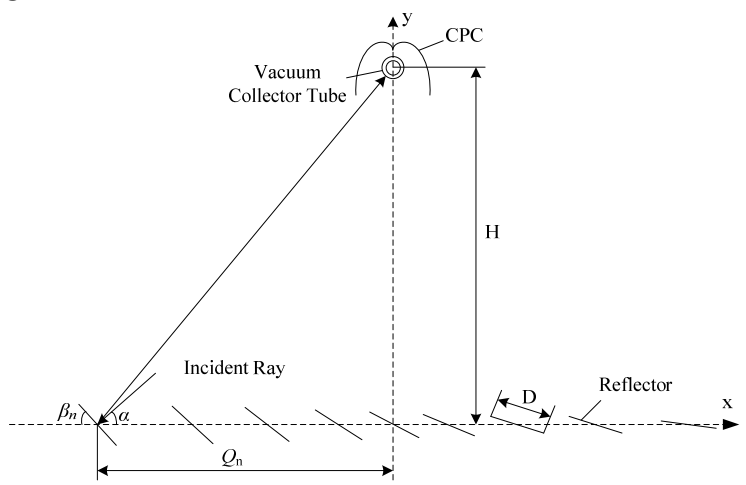

Fig. 1. The schematic of LFR field.

The number of the reflectors of the LFR field is N, which are named $1,2, \ldots$ and $\mathrm{N}$ from left to right. The distance between the N-th reflector and the center of the LFR field is $Q_{n}$. The angle between the n-th reflector and the horizontal plane is $\beta_{n}$ (tracking angle). The reflector width is $D$. The distance between the receiver and the plane of the reflector is $H$.

When the solar incidence angle at a certain time is $\alpha$, the tracking angle $\beta_{n}$ at this time can be calculated via the following expression:

$$
\tan \left(\pi-\alpha-2 \beta_{n}\right)=\frac{H}{Q_{n}}
$$

\section{Shading and blocking influence}

\subsection{Shading and blocking analysis}

The LFR field is symmetric about its centric line. So, shading and blocking analysis is only directed the whole field in morning or afternoon (Real Solar Time), or half of the field in all day. In this paper, the whole field which is arranged in a south-north direction in morning is taken as an example to analyze. This method is also applicable for the LFR field which is arranged in an eastwest direction.

Let coordinate equation of the $n$-th reflector is:

$$
y=k_{n} \cdot x+b_{n}
$$

where, $k_{n}=\tan \beta_{n}, \quad b_{n}=-k_{n} \cdot Q_{n}$.

Let coordinates of left and right endpoints of the n-th reflector are $\left(A_{n}, B_{n}\right)$ and $\left(A_{n}^{\prime}, B_{n}^{\prime}\right)$, then

$$
\begin{gathered}
A_{n}=Q_{n}-\frac{D}{2} \cdot \cos \beta_{n} \\
B_{n}=k_{n} \cdot A_{n}+b_{n} \\
A_{n}^{\prime}=Q_{n}+\frac{D}{2} \cdot \cos \beta \\
B_{n}^{\prime}=k_{n} \cdot A_{n}^{\prime}+b_{n}
\end{gathered}
$$

Shading occurs when one reflector blocks the incident sun rays falling on another reflector. In morning, only the reflector on the east can block the incident sun rays falling on the adjacent reflector on the west.

Let coordinate equation of the incident sun ray passing through the left endpoint of the $n$-th reflector is:

$$
y=k i \cdot x+b i_{n}
$$

where, $k i=\tan \alpha, b i_{n}=A_{n}-k i_{n} \cdot B_{n}$.

A point is found in solving the simultaneous equation group of expressing the sun ray passing through the left endpoint of the reflector on the east and the adjacent reflector:

$$
\left\{\begin{array}{l}
x_{n}=\frac{b_{n}-b i_{n+1}}{k i-k_{n}} \\
y_{n}=k i \cdot x_{n}+b i_{n+1}
\end{array}, 1 \leq n \leq(N-1)\right.
$$

If this point is not on the $\mathrm{n}$-th reflector, there is no shading influence; or shading influence occurs. And shading length $L s h_{n}$ is the distance between the point and the right endpoint of the $\mathrm{n}$-th reflector:

$$
L s h_{n}=\sqrt{\left(A_{n}^{\prime}-x_{n}\right)^{2}+\left(B_{n}^{\prime}-y_{n}\right)^{2}}, 1 \leq n \leq(N-1)
$$

\subsubsection{Blocking analysis}

Blocking occurs when one reflector blocks the reflected rays from another reflector directed towards the receiver. In morning, only the reflector near the center of the LFR field can block the adjacent reflector.

\section{(1) Field on the west of the receiver}

Let coordinate equation of the reflected ray passing through the left endpoint of the $n$-th reflector is:

$$
y=k r_{n} \cdot x+b r_{n}
$$

where, $k r_{n}=\frac{h}{Q_{n}}, b r_{n}=A_{n}-k r_{n} \cdot B_{n}$.

A point is found in solving the simultaneous equation group of expressing the sun ray passing through the left endpoint of the reflector near the center of the LFR field and the adjacent reflector:

$$
\left\{\begin{array}{l}
x_{n}=\frac{b_{n}-b r_{n+1}}{k r_{n+1}-k_{n}} \\
y_{n}=k r_{n+1} \cdot x_{n}+b r_{n+1}
\end{array},\right.
$$

if $\mathrm{N}$ is an even number, $1 \leq n \leq \frac{N-2}{2}$;

if $\mathrm{N}$ is an odd number, $1 \leq n \leq \frac{N-1}{2}$

If the point is not on the n-th reflector, there is no blocking influence; or blocking influence occurs. And blocking length $L b l_{n}$ is the distance between the point and the right endpoint of the $n$-th reflector:

$$
L b l_{n}=\sqrt{\left(A_{n}^{\prime}-x_{n}\right)^{2}+\left(B_{n}^{\prime}-y_{n}\right)^{2}},
$$


if $\mathrm{N}$ is an even number, $1 \leq n \leq \frac{N-2}{2}$;

if $\mathrm{N}$ is an odd number, $1 \leq n \leq \frac{N-1}{2}$

\section{(2) Field on the east of the receiver}

Let coordinate equation of the reflected ray passing through the right endpoint of the $\mathrm{n}$-th reflector is:

$$
y=k r_{n} \cdot x+b r_{n}
$$

where, $k r_{n}=-\frac{H}{Q_{n}}, b r_{n}=y 2_{n}-k r_{n} \cdot x 2_{n}$.

A point is found in solving the simultaneous equation group of expressing the sun ray passing through the right endpoint of the reflector near the center of the LFR field and the adjacent reflector.

$$
\left\{\begin{array}{l}
x_{n}=\frac{b_{n}-b r_{n-1}}{k r_{n-1}-k_{n}} \\
y_{n}=k r_{n-1} \cdot x_{n}+b r_{n-1}
\end{array}\right.
$$

if $\mathrm{N}$ is an even number, $\frac{N+4}{2} \leq n \leq N$;

if $\mathrm{N}$ is an odd number, $\frac{N+3}{2} \leq n \leq N$

If the point is not on the n-th reflector, there is no blocking influence; or blocking influence occurs. And blocking length $L b l_{n}$ is the distance between the point and the left endpoint of the $n$-th reflector:

$$
L b l_{n}=\sqrt{\left(A_{n}-x_{n}\right)^{2}+\left(B_{n}-y_{n}\right)^{2}},
$$

if $\mathrm{N}$ is an even number, $\frac{N+4}{2} \leq n \leq N$;

if $\mathrm{N}$ is an odd number, $\frac{N+3}{2} \leq n \leq N$

\subsection{Shading and blocking efficiency}

The total length of the shading and blocking is the summation of shading length $L s h_{n}$ and blocking length $L b l_{n}$. When shading and blocking influence coexist, the larger value is taken.

The shading and blocking efficiency is:

$$
\eta_{s b}=1-\frac{L s b}{N \cdot D} \times 100 \%
$$

\section{Calculation example analysis}

According to Puerto Errado 1 solar thermal plant, relevant parameters were as follows. The field arranged equal distance with $0.16 \mathrm{~m}$ in an east-west direction. The reflector length was $800 \mathrm{~m}$. The distance between the center of the vacuum collector tube and the plane of the reflector was $7.4 \mathrm{~m}$. The reflector width was $0.8 \mathrm{~m}$. The number of reflectors was 21 . The reflectors were named $1 \#, 2 \#, \ldots$ and $21 \#$ from south to north. Shading and blocking influence at 6:30 of vernal equinox could be calculated by using the MATLAB software. Numerical results were shown in Table 1 respectively.

Table 1. Shading and blocking influence at 6:30 of the vernal equinox.

\begin{tabular}{|c|c|c|c|c|c|c|c|c|c|c|c|c|}
\hline Reflectors (\#) & 1 & 2 & 3 & 4 & 5 & 6 & 7 & 8 & 9 & 10 & 11 & 12 \\
\hline Lsh $(\mathrm{mm})$ & 85.84 & 87.04 & 87.72 & 87.64 & 86.42 & 83.62 & 78.67 & 70.95 & 59.88 & 45.02 & 26.18 & 3.56 \\
\hline$L b l(\mathrm{~mm})$ & 210.50 & 173.33 & 132.83 & 89.01 & 42.19 & 0 & 0 & 0 & 0 & 0 & 0 & 0 \\
\hline
\end{tabular}

Besides, the SolTrace software, which is based on ray tracing method for establishing the model and analyzing the performance of solar power station's concentrator system, was used to simulate the solar thermal plant model to verify the calculation correctness.

The shading occurred on the reflector $2 \#, 5 \#, 8 \#$ and $11 \#$ as shown in Fig. 2. And the blocking occurred on the reflector 2\# and 4\# as shown in Figure Fig. 3.

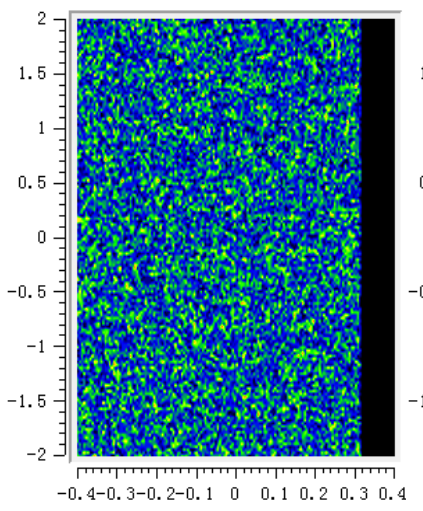

a

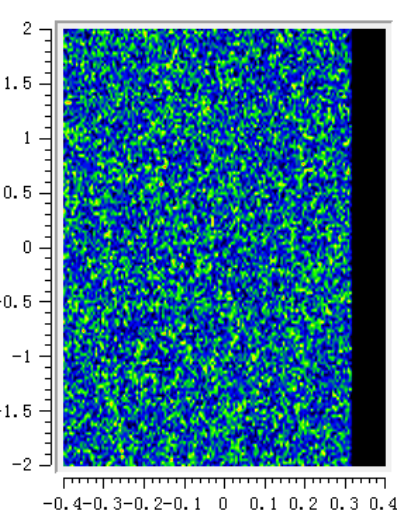

b 


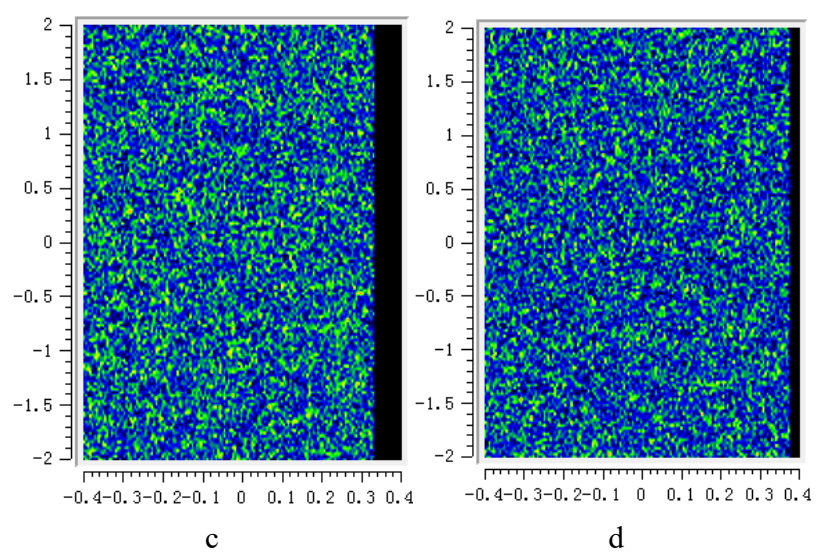

Fig. 2. Shading influence on the reflector $2 \#, 5 \#, 8 \#$ and $11 \#$.

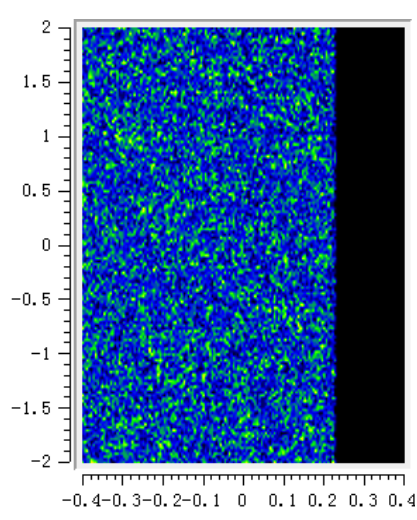

a

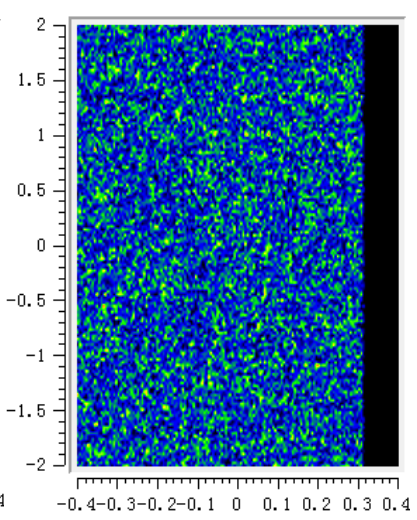

$\mathrm{b}$
Fig. 3. Blocking influence on the reflector $2 \#$ and $4 \#$.

From Fig. 2 and Fig. 3, we can see the shading lengths on the reflector $2 \#, 5 \#, 8 \#$ and $11 \#$, and the blocking lengths on the reflector $2 \#$ and $4 \#$ were good agreement with the results of the calculations in this paper.

In the paper, dynamic analysis of the shading and blocking efficiency all day had been worked out. The shading and blocking efficiencies on the vernal equinox, summer solstice and winter solstice were changing with time as shown in Fig. 4.

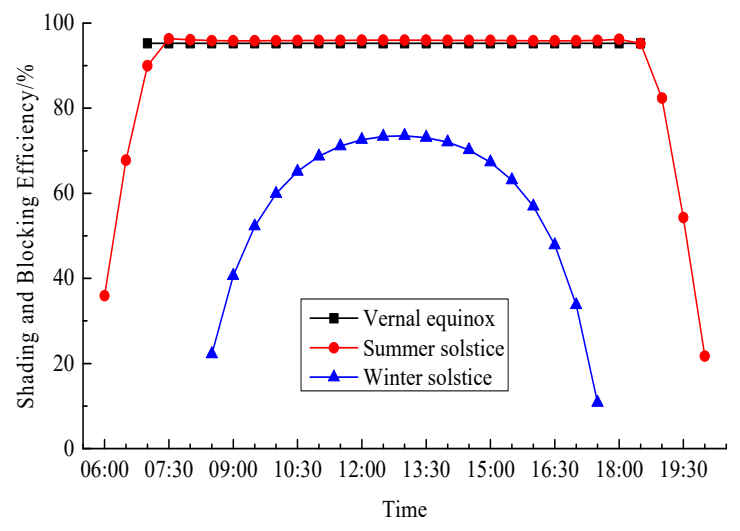

Fig. 4. The shading and blocking efficiencies on the vernal equinox, summer solstice and winter solstice.

As seen in Fig. 4, the rule that the shading and blocking efficiencies change with time on a typical day was good agreement with the results of reference [15]. The shading and blocking efficiencies on vernal equinox hardly changed; on the summer solstice, they were smaller at sunrise and sunset, and gradually stable and close to the value of spring equinox with the increase of solar altitude angle; they were smaller on the winter solstice and the change with time was larger.

\section{Conclusion}

The results of the calculations were good agreement with the simulation dates of SolTrace, and validity of the method was proved. For the Puerto Errado 1 solar thermal plant, the shading and blocking efficiencies on vernal equinox hardly changed; on summer solstice, they were smaller at sunrise and sunset, and gradually stable and close to the value of spring equinox with the increase of solar altitude angle; they were smaller on winter solstice and the change with time were larger.

The study is supported by the Research Project of Institution of Higher Learning of Gansu Province (Grant No.2018A-029) and the Young Scholars Science Foundation of Lanzhou Jiaotong University (Grant No. 2016007).

\section{References}

1. S. Sarkar, and V. Ajjarapu. IEEE Transactions on Sustainable Energy 2, 4 (2011)

2. T. R. Cook, D. K. Dogutan, S. Y. Reece, Y. Surendranath, T. S.Teets, and D. G. Nocera. Chemical Reviews 110, 11 (2010)

3. M. Li, Y. Y. Feng, E. Z. Liu, C. Q. Qin, and W. Feng. Science China 59, 9 (2016)

4. D. Mills. Solar Energy 76(1-3 (2004)

5. J. A. Duffie, and W. A. Beckman. Hoboken, FL: John Wiley \& Sons. (2013)

6. Kalogirou, S. A. Progress in Energy \& Combustion Science 30, 3 (2004)

7. Cavallaro, F. Paper presented at the 13th International Symposium on "Concentrating Solar Power and Chemical Energy Technologies", Seville, Spain, June 20-23 (2006)

8. Gharbi, N. E., H. Derbal., S.Bouaichaoui, and N. Said. Energy Procedia 6, 6 (2011)

9. Qiu, Y., Y. L. He, Z. D. Cheng, and K. Wang. Applied Energy 146 (2015)

10. Grena, R., and P. Tarquini. Energy 36, 2 (2011)

11. Abbas, R., J. Muñoz-Antón, M. Valdés, and J. M. Martínez-Val. Energy Conversion \& Management 72, 4 (2013)

12. Yellowhair, J., and J. D. Ortega. Paper presented at the International Society for Optics and Photonics, September 16 (2014)

13. Budin, R., and L. Budin. Solar Energy 29, 4 (1982)

14. Niewienda, A., and F. D. Heidt. Solar Energy 58, 4-6 (1996)

15. Du, C. X. , P. Wang, Y. T. Wu, C. F. Ma, and H. Guo. Acta Energiae Solaris Sinica 34, 8 (2013) 